\title{
A recent merger in the Fornax dwarf spheroidal galaxy
}

\author{
Matthew G. Coleman \\ Research School of Astronomy and Astrophysics, Mount Stromlo Observatory, Cotter Road, \\ Weston, ACT, 2611, Australia \\ email: coleman@mso.anu.edu.au
}

\begin{abstract}
This contribution describes a recent photometric survey of the Fornax dwarf spheroidal galaxy. CCD photometry in two colours $(V$ and $I)$ was obtained for a 10 degree $^{2}$ region centred on Fornax. By selecting stars sharing the colour-magnitude space of this system's RGB, we were able to remove the majority of field stars, and thereby probe the structure of Fornax at a previously unattained level. The data indicate that this dwarf galaxy contains two shells, both aligned with the Fornax major axis. Also, two 'lobed' structures sharing the alignment of these shells were discovered beyond the nominal tidal radius. These structures appear to be the remnant of a companion dwarf galaxy which merged with Fornax approximately 2 Gyr ago.
\end{abstract}

Keywords. galaxies: dwarf — galaxies: individual (Fornax) — galaxies: photometry — galaxies: stellar content — galaxies: interactions — Galaxy: halo

\section{Introduction}

Observations of large-scale structure throughout the Universe indicate galaxies are arranged in clusters and filaments, surrounding large voids which contain few luminous objects. The processes leading to this structure have been investigated by dark matterdominated cosmological simulations which predict that a complex system of hierarchical merging formed the galaxies of the present day. Evidence of these mergers can be seen as remnant structure in large spiral and elliptical galaxies. Malin \& Carter (1980) detected phase-wrapped shells in the haloes of large galaxies (Malin 1978), and found that such structure is relatively common (Malin \& Carter 1983). Simulations by Hernquist \& Quinn (1988), Hernquist \& Quinn (1989) indicate that these shells originated through the merging of a smaller companion galaxy with its host object. Hence shell structure is a common remnant of large galaxy mergers.

If such structure can be seen in large galaxies, it is reasonable to expect a similar effect in smaller bodies such as the dwarf galaxies. Evidence of recent mergers in dwarf galaxies has not previously been observed. In this contribution we summarise the results presented in Coleman et al. (2004; hereafter C04) and Coleman et al. (2005; hereafter C05). These analyses have found the structure of Fornax to be surprisingly complex. The system contains two shell-like structures aligned with the major axis, one located near the core radius, and the other well beyond the nominal tidal radius. Additionally, two stellar extensions have been found to either side of Fornax, also aligned with the two shells. We inferred that these structures are the remnants of a companion dwarf galaxy which merged with Fornax approximately 2 Gyr ago.

\section{Initial Analysis - The South-East Shell}

Stetson, Hesser, \& Smecker-Hane (1998) describe a deep survey of the central regions of Fornax. They were able to cover the inner $1 / 3 \mathrm{deg}^{2}$ of the system, out to $\sim 2$ core 

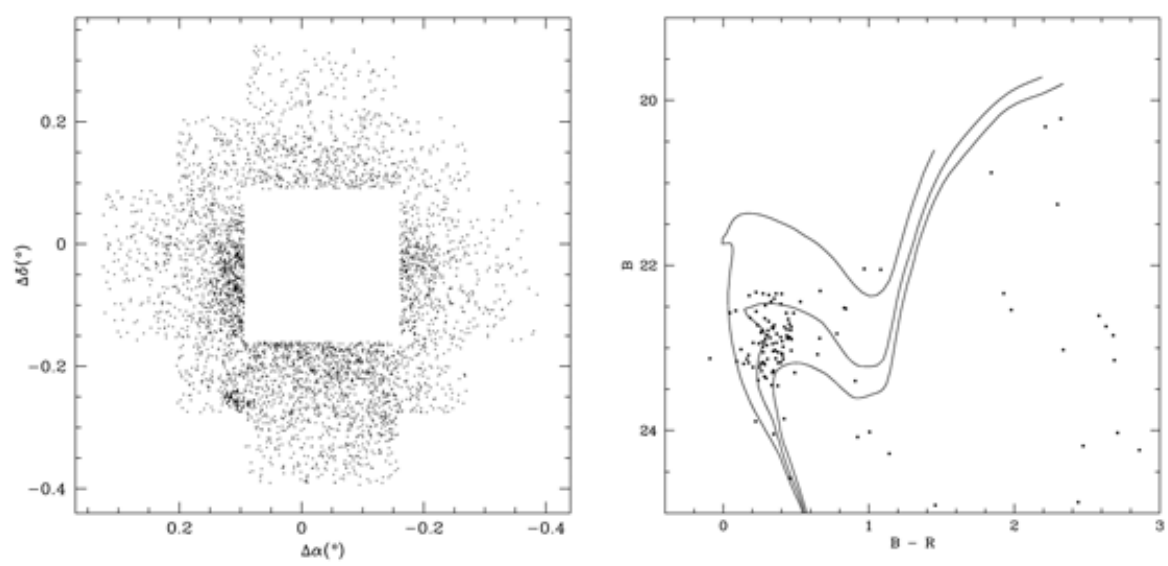

Figure 1. Left panel: The spatial distribution of young main sequence stars. The overdense region is clearly visible in the lower left of the figure. The central regions of Fornax experience substantial inter-field completeness variations at the selected colours and magnitudes, and have been excluded. Right panel: Subtracted CMD for the overdense region constructed using a Monte Carlo subtraction technique. Overplotted are Yonsei-Yale isochrones for ages of 1, 2 and 3 Gyr, and an assumed metallicity $[\mathrm{Fe} / \mathrm{H}]=-1.0$.

radii. Stetson et al. (1998) found a substantial number of young main sequence stars (age $\sim 100 \mathrm{Myr}$ ), indicating that star formation in Fornax has continued almost to the present day. Also, the distribution of these young stars is complex, possibly indicating some dynamical process was involved in their formation.

We obtained the Stetson et al. (1998) data and re-analysed the distribution of these young stars (C04). This is shown in Fig. 1, and immediately visible is a shell-like structure located 17 arcmin $(\sim 1.8$ core radii) from the centre of the system. By subtracting the background population, it was found that this structure is composed almost exclusively of a stellar population with an age of 2 Gyr; see Fig. 1. It is aligned with the major axis of Fornax, and is situated on the minor axis. Our inference was that the stellar association represents shell structure in a dwarf galaxy, the remnant of a merger between Fornax and a gas-rich companion approximately 2 Gyr ago. However, such a conclusion was based on a single shell located towards the central regions of Fornax. Thus, our aim was to conduct a photometric survey across the entire Fornax body, and to determine if the outer regions of the system contained similar structures.

\section{The Fornax Survey}

We collected photometry in $V$ and $I$ bands for an area of $3.1 \times 3.1 \mathrm{deg}^{2}$ centred on the Fornax dSph using the Wide-Field Imager (WFI) attached to the SSO 1 metre telescope. A description of the data analysis is presented in C05. By selecting stars within the RGB selection range, we are able to increase the 'signal' of Fornax stars compared to the background field population (the 'noise'). We selected candidate RGB stars down to the completeness limit, and their distribution is shown in the left panel of Fig. 2. The outer red ellipse traces the nominal tidal radius of this system, taken from Mateo (1998). The right panel contains a contour plot of Fornax, where each star have been convolved with a Gaussian function.

Perhaps the most apparent feature in Fig. 2 is a region of increased stellar density located approximately $1.3^{\circ}$ north-west from the centre of Fornax. We have measured 
the total surface density in the vicinity of the feature to be $1.3 \pm 0.1$ times that of the remainder of F4. That is, at least one quarter of the stars in the overdense region are part of the feature, while the remainder form the background population. We removed the background population and, assuming the feature lies at the same distance modulus as Fornax $[(m-M)=20.70$; Mateo 1998], then it has an integrated absolute magnitude of $M_{V}=-6.7 \pm 0.2$. Additionally, two 'lobe' structures are visible in the contour plot. Similar to the two shells, these lie on the Fornax minor axis.

\section{Analysis}

A full description of the analysis techniques are presented in C05. We applied two techniques to measure the level of stellar clustering beyond the tidal radius. The first technique resulted in a function which described the probability of measuring a given density in the extra-tidal region. This provided a significance limit on the north-western shell and the two lobed structures on the minor axis. Secondly, we applied the two-point angular correlation function, which is commonly used to measure the level of galaxy clustering in cosmological studies.

From the probability density function, it was found that the extra-tidal region consists of three populations with distinguishable densities. The north-western shell was a $\sim 20 \sigma_{\mathrm{f}}$ result, where $\sigma_{\mathrm{f}}$ is the standard deviation in the density of the field population. The density of the two lobes was measured to be separated from that of the field population by $4.5 \sigma_{\mathrm{f}}$, implying they are not due to random fluctuations in the field star distribution. Assuming they are at the same distance as Fornax, with a similar stellar population, they measure $\sim 8 \mathrm{kpc}$ in length with an integrated absolute visual magnitude of $M_{V} \sim-9$. The integrated luminosity of these structures imply that the merged companion was at least as bright as a small dwarf galaxy such as Ursa Minor or Draco.

\section{Discussion}

The detected structures provide a strong argument for a recent merger in the Fornax system. However, another possibility is that the two large lobes of material extending to either side of the dSph represent tidal tails. They qualitatively match simulations of tidal tails (Helmi \& White 2001; Mayer et al. 2001) which are caused by interaction with the Milky Way. These models also indicate that material leaves the satellite in bursts, which may produce the observed shell-like structure.

However, clear tidal tails have only been observed in a single dSph satellite of the Galaxy; the Sagittarius dSph. The Sagittarius orbit brings it within approximately 15 $\mathrm{kpc}$ from the Galactic centre with an orbital eccentricity of $e \approx 0.75$ (Law, Johnston, \& Majewski 2004), and thus Sgr experiences substantial tidal forces. In contrast, Fornax is located at a distance of $140 \mathrm{kpc}$ (Mateo 1998) and proper motion measurements indicate it is on a polar orbit with a small eccentricity $(e=0.27)$ and is currently at perigalacticon (Dinescu et al. 2004). Moreover, Fornax contains a mass larger than all the other Galactic dSphs combined (excluding Sagittarius), which further precludes the possibility of stars escaping from this relatively deep potential well. Thus, given the relatively large mass, distance and orbital parameters of Fornax, we do not expect to observe such clear evidence of Milky Way interaction.

Further, the inner shell described in C04 demonstrated a clear signature; it was dominated by stellar population with an age of $2 \mathrm{Gyr}$, significantly different to the mixed stellar population observed in Fornax (Stetson et al. 1998). If the observed substructure 



Figure 2. Left panel: Distribution of RGB-selected stars on the sky. The inner and outer ellipses are the core and tidal radii respectively (Mateo 1998). Right panel: Distribution of Fornax RGB stars where each star has been convolved with a Gaussian of radius $40^{\prime \prime}$. The contours are logarithmically spaced, and are fitted with a smoothing length of $3.0^{\prime}$. The first contour represents a stellar density $3.3 \sigma_{\text {field }}$ above the field star population.

is due to the gravitational influence of the Galaxy, then it would contain the same stellar population as the remainder of the system.

The high density structure located $1.3^{\circ} \mathrm{NW}$ of Fornax is clearly shell-like in appearance. In the case of shell structure, the lobes would represent those portions of shell for which we do not see a limb brightening effect; in essence, they lie in front of, or behind, the Fornax system. These appear remarkably similar to the simulations of interacting dark halos presented by Knebe et al. (2005). To further investigate the shell possibility, we will obtain deep photometry of the shell region. If the stellar population is similar to the Fornax system, this would indicate the substructures are tidal tails. However, if the stars in the shell display a marked age difference from those in Fornax, this would support the possibility of shell structure in a dSph.

\section{References}

Coleman, M., Da Costa, G.S., Bland-Hawthorn, J., Martínez-Delgado, D., Freeman, K.C. \& Malin, D. 2004, AJ 127, 832 (C04)

Coleman, M.G., Da Costa, G.S., Bland-Hawthorn, J. \& Freeman, K.C. 2005, AJ 129, 1443 (C05)

Dinescu, D.I., Keeney, B.A., Majewski, S.R. \& Girard, T.M. 2004, AJ 128, 687

Helmi, A.\& White, S.D.M. 2001, MNRAS 323, 529

Hernquist, L.\& Quinn, P.J. 1988, ApJ 331, 682

Hernquist, L.\& Quinn, P.J. 1989, ApJ 342, 1

Knebe, A., Gill, S.P.D., Kawata, D. \& Gibson, B.K. 2005, MNRAS 357, L35

Law, D.R., Johnston, K.V. \& Majewski, S.R. 2005, ApJ 619, 807

Malin, D.F. 1978, Nature 276, 591

Malin, D.F. \& Carter, D. 1980, Nature 285, 643

Malin, D.F. \& Carter, D. 1983, ApJ 274, 534

Mateo, M. 1998, ARA\&A 36, 435

Mayer, L., Governato, F., Colpi, M., Moore, B., Quinn, T., Wadsley, J., Stadel, J. \& Lake, G. 2001, ApJ 559, 754

Stetson, P. B., Hesser, J. E. \& Smecker-Hane, T. A. 1998, PASP 110, 533 


\section{Discussion}

SkILlman: Why do you favour a two-lobe interpretation over a spherical halo?

Coleman: The two observed shells support a merger scenario where the companion approached on a radial vector along the minor axis. A two-lobe structure is a natural consequence of such a merger, however further observations may reveal a spherical shell system.

CONSELICE: Can you rule out the possibility that Fornax is being tidally stripped, which could explain these observations?

Coleman: Not conclusively. However, the large mass, distance and relatively gentle orbit of Fornax compared to the other Galactic dSphs make such tidal structures highly improbable.

VADUVESCU: I could not understand how you subtracted the foreground stars.

Coleman: Briefly, using Monte Carlo simulations using stars in a shell around the galaxy.

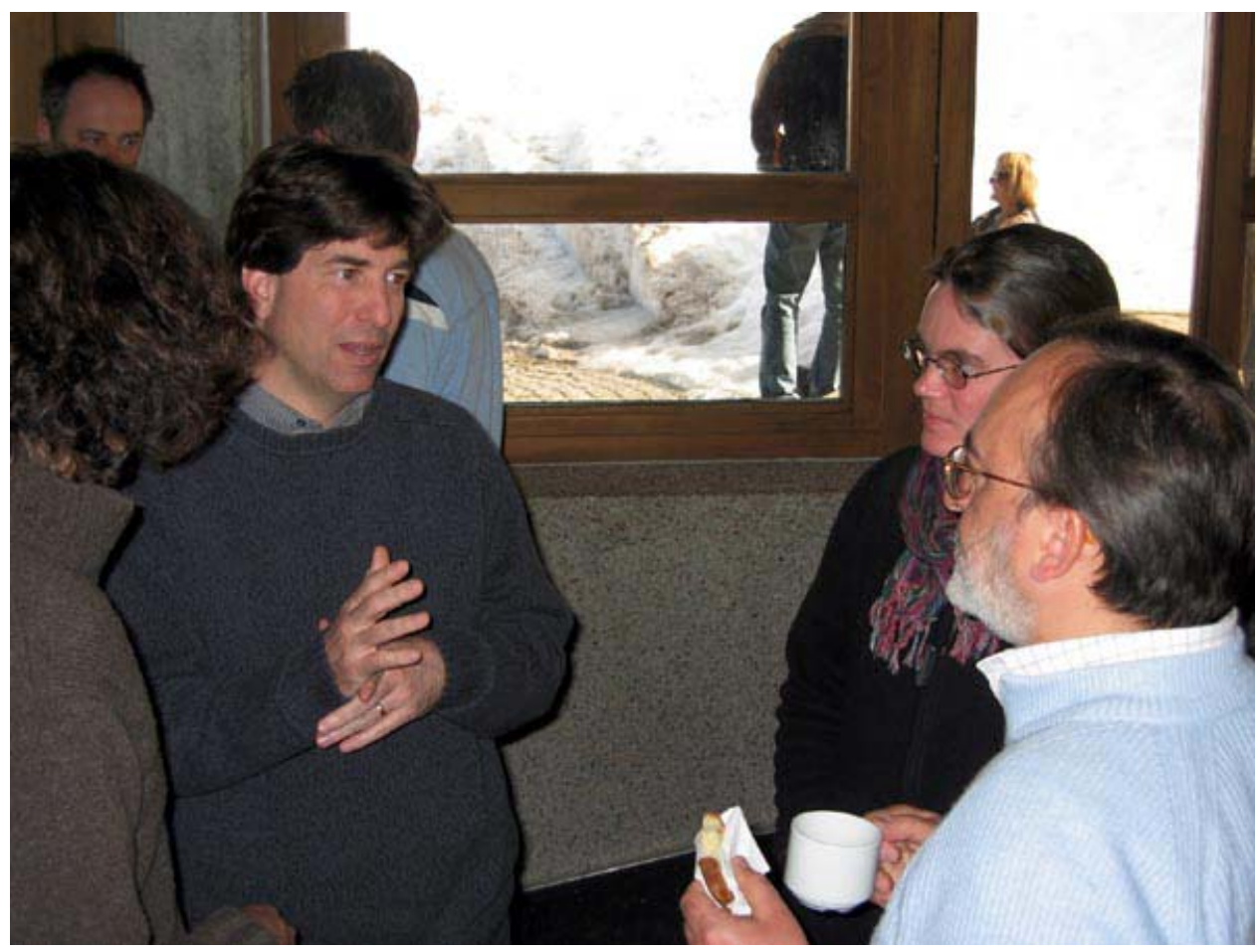

